\title{
Diagnostic value of cytological and microbiological methods in cryptococcal meningitis
}

\author{
M. Zhang ${ }^{1 *}$, J.C. Li ${ }^{2 *}$, H. Lin ${ }^{1}$, W. Zhang ${ }^{2}$, M. Lin ${ }^{1}$, L. Wu ${ }^{1}$, W. Liu ${ }^{3}$, \\ J.S. Mu' ${ }^{1}$ J.X. Ye ${ }^{1}$ and X.P. Cui ${ }^{1}$ \\ ${ }^{1}$ Department of Neurology, Fuzhou Dongfang Hospital, \\ Fujian Medical University, Fuzhou, China \\ ${ }^{2}$ Department of Emergency Medicine, Fuzhou Dongfang Hospital, \\ Fujian Medical University, Fuzhou, China \\ ${ }^{3}$ Department of Pathology, Fuzhou Dongfang Hospital, \\ Fujian Medical University, Fuzhou, China \\ *These authors contributed equally to this study. \\ Corresponding author: J.C. Li \\ E-mail: jingchenglicn@yeah.net / lijingcheng7510@sina.cn
}

Genet. Mol. Res. 13 (4): 9253-9261 (2014)

Received April 30, 2013

Accepted October 22, 2013

Published March 26, 2014

DOI http://dx.doi.org/10.4238/2014.March.26.3

\begin{abstract}
The aim of this study was to investigate diagnostic methods for cryptococcal meningitis (CM). A retrospective analysis was conducted for 31 patients with CM confirmed by etiologic detection of cerebrospinal fluid in our hospital in the past 5 years. Nineteen cases in 31 patients were confirmed with $\mathrm{CM}$ in the first diagnosis, with a misdiagnosis rate of $38.7 \%$. The positive rates of cryptococcus detection in cerebrospinal fluid with May-GrünwaldGiemsa (MGG)-, ink-, and Alcian blue-staining methods were $86.9,70.9$, and $80.6 \%$, respectively. The misdiagnosis rate of CM is high during the early stage of disease. The total positive rate of cryptococcus diagnosis using the MGG-staining method was significantly higher than that using the ink-staining method. These
\end{abstract}


results are important for diagnosing CM.

Key words: Cryptococcal meningitis; Cerebrospinal fluid; Ink staining; Diagnostic methods; MGG staining; Alcian blue staining

\section{INTRODUCTION}

Cryptococcal meningitis $(\mathrm{CM})$ is a subacute or chronic inflammatory disease of the central nervous system (CNS) caused by the meninges infection with Cryptococcus neoformans. A few CM cases show intracranial space-occupying lesions (Whyte and Eshkar, 2007; Chang et al., 2008; Pukkila-Worley and Mylonakis, 2008). C. neoformans is widely distributed in nature, particularly in pigeon excreta, which is an important infection source. It can also be isolated from the body surface, oral cavity, and human fecal matter. The thallus of $C$. neoformans is circular, with a diameter of 4-20 $\mu \mathrm{m}$. On its surface, there is a layer of thick hard colloidal film, 1-3 times larger than the thallus. Currently, there are 2 types of cryptococcus with pathogenicity to humans, including C. neoformans and Cryptococcus gattii. As conditional yeast with a capsular, C. neoformans is the most common fungal medium causing CNS diseases (Jain et al., 2007). This species can be divided into 4 serotypes: $\mathrm{A}, \mathrm{B}, \mathrm{C}$, and $\mathrm{D}$, according to antigenicity. Type $\mathrm{A}$ and $\mathrm{D} C$. neoformans are often clinically isolated. These species show global distribution and often infect patients with acquired immunodeficiency syndrome (AIDS). Type B and C C. neoformans are relatively rare. In China, the most common serotype is type A, with a total incidence of $95 \%$ (Lin, 2009). Due to the low clinical incidence and complex clinical manifestation, as well as its atypical characteristics, CM is easily confused with other CNS diseases during early disease stages, resulting in misdiagnosis and mistreatment. Recently, with the development of new medical technologies such as organ transplantation and immunosuppressive therapy, as well as the increase of HIV infection and occurrence of AIDS, the incidence rate of CM has increased (Dammert et al., 2008). Therefore, fast and efficient diagnosis of CM is a key task for nerve physicians.

Identifying cryptococcus is a criterion for diagnosing $\mathrm{CM}$. Commonly used cryptococcus detection methods include: 1) The fungal culture method, which is a gold standard for diagnosing cryptococcosis, but is not practical because it is time-consuming and has a low positive rate. There were no positive cases for fungal culture in this study. 2) An immunological method in which the polysaccharide antigen of $C$. neoformans capsular was tested through a latex agglutination experiment. This method is rapid and effective. As reported by Bicanic and Harrison (2005), the positive rate of detecting the $C$. neoformans antigen using this method is $76 \%$. Xue and Liu (2008) found that the positive rate of $C$. neoformans antigen is $100 \%$. However, this method has no specificity for diagnosing fungal infection, and other diagnostic tests and identification methods should also be conducted. This method can only be used for auxiliary examination. 3) The PCR method (Su and Ma, 1997) involves specific oligonucleotide primers, which are designed according to the conservative sequence of $C$. neoformans, and then the PCR is conducted for rapid and specific detection of C. neoformans. This method is very strict with the PCR system. Mismatch between amplification products and deviation of the amount and proportional of each component can result in false-positive results.

In this study, to improve the diagnostic level of $\mathrm{CM}$ and identify a better detection method for C. neoformans in the laboratory, a retrospective analysis was conducted on 31 adult patients with CM confirmed by etiologic detection of cerebrospinal fluid (CSF) in the Department of Neurology, Fuzhou Dongfang Hospital, from September 2006 to February 2012. 


\section{MATERIAL AND METHODS}

\section{General data}

Thirty-one patients were diagnosed with $\mathrm{CM}$ according to the criterion for identifying cryptococcus in CSF using staining methods (Thwaites et al., 2002). There were 18 males and 13 females in the study aged $19-56$ years, with average age of $43.7 \pm 11$ years. The duration from disease occurrence to clinical diagnosis was 11 days to 5.4 months, with an average duration of $45.3 \pm 11.3$ days. The frequency of CSF detection until identifying cryptococcus was 1-6 times. Twelve patients had contacted the disease from a pigeon or its excreta. Nineteen cases were diagnosed with $\mathrm{CM}$ combined with other systemic diseases, with 3 cases of systemic lupus erythematosus, 7 cases of kidney transplantation, 3 cases of liver transplantation, 2 cases of nephropathy syndrome, 2 cases of diabetes, 1 case of liver cancer, 1 case of breast cancer, 1 case of gastric cancer, and 1 case of AIDS. The cases of transplantation combined with cryptococcal infection accounted for $32.3 \%$ of the total incidence. This study was conducted in accordance with the declaration of Helsinki. This study was conducted with approval from the Ethics Committee of Fuzhou Dongfang Hospital. Written informed consent was obtained from all participants.

\section{Clinical symptoms}

All patients had subacute or chronic disease. There were 29 cases of headache, 22 cases of nausea and vomiting, 28 cases of fever ( 21 cases with high fever with body temperature over $39.1^{\circ} \mathrm{C}$ ), 8 cases of lags in response and significantly decreased memory, 9 cases of decreased visual acuity, 6 cases of hearing loss, 3 cases of convulsions, 4 cases of behavioral and psychological disorders, and 3 cases of disturbance of consciousness. In addition, 27 patients had nuchal rigidity and/or positive meningeal irritation signs, including 8 cases of positive pathologic reflex, 2 cases of hemiplegia and/or paraplegia, and 21 cases of papilledema.

\section{Misdiagnosis}

Nineteen cases were confirmed with $\mathrm{CM}$ in the first diagnosis, with a misdiagnosis rate of $38.7 \%$. Two patients were misdiagnosed with upper respiratory tract infection, 3 cases with viral meningitis, 5 cases with tuberculosis meningitis, and 2 cases with vascular headache.

\section{Experimental methods}

CSF was drawn from the patient by lumbar puncture within 2 days after hospitalization and stained with May-Grünwald-Giemsa (MGG), ink, and Alcian blue. The fungus culture, drug sensitivity test, acid-fast staining, tubercle bacillus culture, and biochemical test were conducted on the CSF sample. CSF in which the cryptococcus was first detected was used for cytological experiments. The staining methods were as follows. The white blood cell (WBC) counting was determined for the CSF sample, after which $0.1-0.5-\mathrm{mL}$ (depended on the number of cells) of the CSF sample was placed into an FMU-5 micro cell glass slide centrifugal precipitator (Su and Kong, 2001) for low-speed centrifugation (500 rpm, 2-5 min). The glass slide was treated with 
MGG staining (10 min), Alcian blue staining (15 min), ink staining (immediate), and WBC classification. For MGG- and Alcian blue-staining methods, the glass slide was dried after staining and optical microscopy (oil microscope) was used to detect cryptococcus and WBC. For the ink staining method, microscopic examination was conducted immediately after staining.

\section{Statistical analysis}

Data are reported as means $\pm \mathrm{SD}$. Statistical analysis was performed using the SPSS 13.0 statistical software. A chi-square test (4-fold table) was used for single-factor analysis. P $<0.05$ was considered to be statistically significant.

\section{RESULTS}

\section{Auxiliary examination}

In 31 patients, there were 25 cases with increased peripheral WBC number, including 17 cases with WBC of $10-15 \times 10^{9} / \mathrm{L}, 5$ cases WBC of $15-20 \times 10^{9} / \mathrm{L}$, and 3 cases with WBC greater than $20 \times 10^{9} / \mathrm{L}$. One case was with positive for the HIV antibody and 28 cases showed an increased blood sedimentation rate. Twenty-seven cases showed abnormal electroencephalogram, including 5 cases with mild abnormalities, 18 cases with moderate abnormalities, 4 cases with severe abnormalities, 6 cases with paroxysmal slow wave, 22 cases with wide range of slow wave and slow wave rhythm, and 1 case with spikes and sharp waves and spike-slow wave rhythm. There was a variety of non-specific MRI manifestations for CM (Ostxow and Hudgins, 1994). In 5 patients with MRI abnormalities, there were 5 cases of meningeal enhancement, 3 cases of ventricle enlargement, and 1 case of meningeal granuloma.

\section{General CSF examination}

In 31 patients, there were 26 cases with increased intracranial pressure, including 17 cases with pressure over $300 \mathrm{mmH}_{2} \mathrm{O}$, and 5 cases with pressure between 60 and $80 \mathrm{mmH}_{2} \mathrm{O}$. Thirty cases showed WBC higher than normal $\left(135 \pm 18 \times 10^{6} / \mathrm{L}\right)$. There were 28 cases with decreased glucose content $(2.1 \pm 1.2 \mathrm{mM})$, and 4 cases with decreased chloride content (119 $\pm 12 \mathrm{mM})$. In all patients, the total protein content increased $(2.8 \pm 0.7 \mathrm{~g} / \mathrm{L})$. Values were negative for mold and Mycobacterium tuberculosis culture, and the acid-fast staining was also negative.

\section{CSF staining}

The results of CSF staining with MGG, ink, and Alcian blue are shown in Figure 1. In 31 patients, 19 cases were confirmed to have $\mathrm{CM}$ at the first diagnosis, with a misdiagnosis rate of $38.7 \%$. The total positive case and total positive rate with MGG, ink, and Alcian blue staining methods were 27 (86.9\%), 22 (70.9\%), and 25 (80.6\%), respectively (Table 1). The positive rates at first detection using the 3 methods were $61.3,41.9$, and $48.4 \%$, respectively (Table 2). The maximum frequency of CSF detection until identifying cryptococcus was 6 times. 


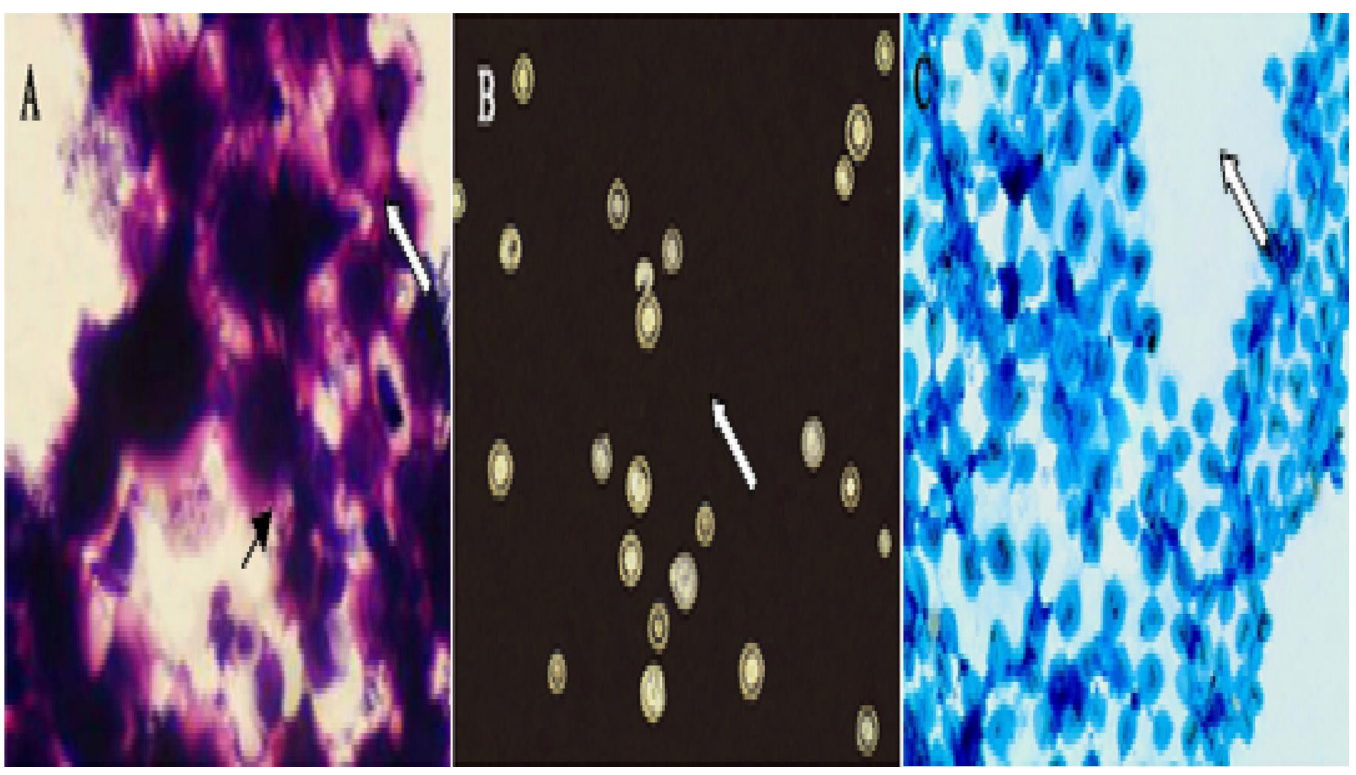

Figure 1. Results of CSF staining (40X). A. MGG staining. B. Ink staining. C. Alcian blue staining. Black arrow $=$ Cryptococcus with deletion of capsule; white arrows = Cryptococcus with intact capsule.

Table 1. Total positive case and total positive rate of the three staining methods.

\begin{tabular}{lcccc}
\hline Staining method & Total positive case & Total negative case & Total case & Total positive rate \\
\hline MGG staining & 27 & 14 & 31 & 86.9 \\
Ink staining & 22 & 9 & 31 & $70.0^{*}$ \\
Alcian blue staining & 25 & 16 & 31 & $80.6^{*}$ \\
\hline
\end{tabular}

$* \mathrm{P}<0.05$.

Table 2. Positive case and positive rate in the first detection of the three staining methods.

\begin{tabular}{lcccc}
\hline Staining method & Positive case & Negative case & Total case & Positive rate \\
\hline MGG staining & 19 & 12 & 31 & 61.3 \\
Ink staining & 13 & 18 & 31 & $41.9^{*}$ \\
Alcian blue staining & 15 & 16 & 31 & $48.4^{*}$ \\
\hline
\end{tabular}

$* \mathrm{P}<0.05$.

\section{Cytological characteristics of CSF}

In 31 patients, CSF sample in which the cryptococcus was first detected was used for cytological experiments. Results are shown in Figure 2. The earliest and latest time at which the cryptococcus was detected included the 11th and 165th days after disease occurrence, respectively. The lymphocyte reaction was the main WBC classification characteristic of CSF. Cell counting results showed that the proportion (median) of lymphocyte, neutrophils, activated lymphocyte, monocyte, phagocyte (phagocytic leukocyte, and phagocytic cryptococcus), eosinophil, and plasmocyte was $41,18,7,27,1,4.5$, and $0.5 \%$, respectively. 


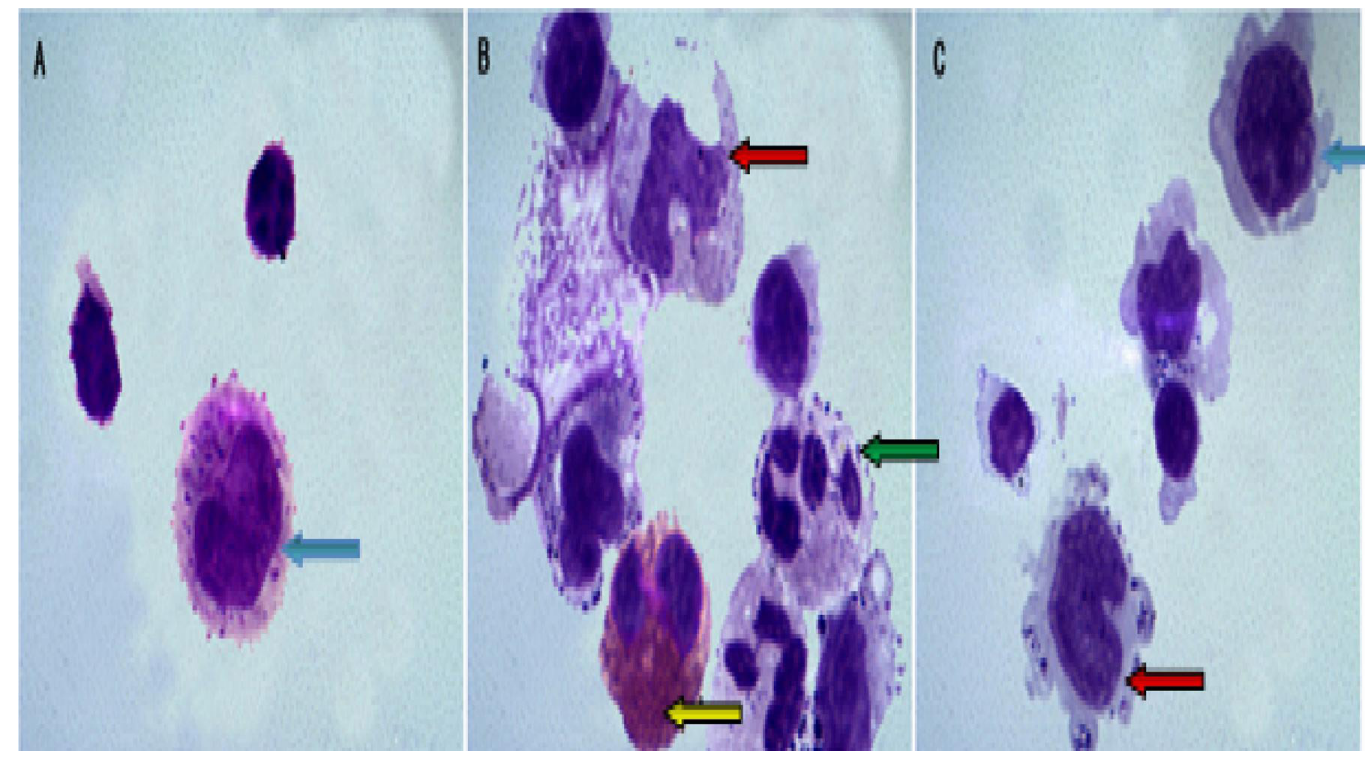

Figure 2. Cytological characteristics of CSF (100X). A. CSF in normal human; B. CSF in patients with CM 1 month after disease occurrence; C. CSF in patients with disease improvement. Blue arrows = lymphocyte; red arrows $=$ monocyte; green arrow $=$ neutrophil; yellow arrow $=$ eosinophil.

\section{DISCUSSION}

CM is the most common CNS cryptococcosis. Previous studies have shown that $85 \%$ of patients with CM also have HIV and AIDS in Europe and the United States. In China, because of long-term, widespread use of broad-spectrum antibiotics, adrenal cortical hormone and immunosuppressants, and the development and popularization of organ transplantation technologies, the incidence of CM had increased markedly, resulting in 25-80\% morbidity of fatal systemic cryptococcosis (Juhi et al., 2009). The incidence rate of cryptococcosis is $2.8 \%$ in patients who have undergone organ transplantation (Husain et al., 2001; Singh et al., 2008a). Sixty percent of patients with cryptococcosis have CNS involvement (Singh et al., 2008b), and the mortality rate of cryptococcosis is close to $50 \%$ (Vilchez et al., 2002). In the 31 patients in our study, CM incidence showed an increasing trend by year. The morbidity in patients with organ transplantation combined with central cryptococcosis was up to $32.3 \%$. The main reasons for cryptococcus susceptibility in the CNS may be that there is no cryptococcus antibody in the CSF, and CSF nutrients are the most suitable for cryptococcus growth and reproduction. Other studies suggest that the dopamine melanin secreted by the hypothalamus is conducive to cryptococcus growth.

Currently, the lack of sensitive, specific etiological detection facilities and clinical specificity result in CM misdiagnosis and mistreatment. In this study, 12 cases of 31 patients were misdiagnosed in the first diagnosis, with a misdiagnosis rate of $38.7 \%$. The main reasons may be as follows: 1) The typical clinical manifestation and specific auxiliary examination results are lacking. CM is easily confused with other CNS infectious diseases. 2) Most patients are accompanied by other underlying diseases with low immunity and unobvious neurological 
symptoms. 3) CM symptoms are concealed after hormone treatment. 4) The detection method is not sufficiently sensitive to detect cryptococcus in the CSF. 5) Intracranial pressure cannot be used as standard for diagnosing CM. In previous study (Macsween et al., 2005), intracranial pressure significantly increased before systemic antifungal therapy, but there was no obvious change in 5 cases of 31 patients in this study. The lowest intracranial pressure was $60 \mathrm{mmH}_{2} \mathrm{O}$.

In this study, CSF staining methods were used to diagnose CM. Their respective advantages and disadvantages are described below.

\section{Ink staining}

This simple, rapid method is widely used in general hospitals, but low CSF amounts and the requirement for repeated examination are disadvantages. The positive rate with first ink staining is $58.1 \%$, and for consecutive repeat staining only $53-56 \%$. In this study, the positive rate with first staining was $48.4 \%$, with a total positive rate of $70.9 \%$. The reasons for the low positive rate may be that the small amount of CSF results in misdiagnosis. A small amount of cryptococcus is misdiagnosed as WBCs or red blood cells. The cryptococcus with capsule deletion or variety cannot be stained.

Additionally, the time required for staining or detection is long.

\section{Alcian blue staining}

Cryptococcus can be stained using Alcian blue because of the large amount of acidic mucopolysaccharide involved in its capsular. After staining, the capsular is dark blue and the thallus is light blue, while the surrounding inflammatory cells are not colored. Thus, the staining is very clear, even with low amounts of cryptococcus. The positive rate is approximately $80 \%$ (He and $\mathrm{He}, 2007$ ). In this study, the positive rate at first detection was $67.7 \%$, with a total positive rate of $80.6 \%$. The running off of CSF, misdiagnosis for a small amount of cryptococcus, and non-staining of cryptococcus with deletion of capsular are the main causes of false-negative results.

\section{MGG staining}

This method has the advantages of retaining the intact cell morphology, accurate classification, simple operation, easy observation, and low misdiagnosis rate. The reported positive rate of this method is up to $94 \%$ ( $\mathrm{Li}$ and $\mathrm{Lin}, 2004$ ). It can detect very small amounts of cryptococcus in initial CSF samples, which cannot be detected using the ink-staining method. This has greatly improved the detection level. This method is suitable not only for cryptococcus with intact capsular, but also for cryptococcus with deleted capsular (Figure 1A). Thus, the positive rate of diagnosis is greatly enhanced. In this study, the positive rate with first staining was $61.3 \%$, with a total positive rate of $86.9 \%$. The first positive rate was significantly higher than that of the ink- and Alcian blue-staining methods, respectively. The total positive rate was also significantly higher than ink staining.

Although the classification method of WBC in CSF cannot be used for directly diagnosing CM, it plays an important auxiliary role in diagnosis. The WBCs in CSF of normal humans consist of lymphocytes and monocytes at a ratio of $6: 4$ or 7:3. There are a small 
number of neutrophils $(<2 \%)$ in the CSF of some people. During the early and middle stages of CM, the mixed cell reaction is dominant in CSF. In addition to lymphocytes, monocytes, activated lymphocytes, and activated monocytes, increased neutrophils also exist, accompanied by more eosinophils and a few plasmocytes and mononucleophages, particularly eosinophils, the proportion of which markedly increases compared to patients with other meningitis types. In this study, the mixed cell reaction was dominant in 30 cases of 31 patients, with neutrophil and eosinophil proportions of 51 and $11 \%$, respectively. The median was 18 and $4.5 \%$, respectively (Figure 2B). There were 17 cases $(54.8 \%)$ with mixed lymphocyte reactions. With the extension of the disease course, CM symptoms were gradually reduced, with decreasing pathological cell numbers (Figure 2C).

The MGG-staining method has the advantages such as simple operation and a low amount of time required. Additionally, the unique morphological features of cryptococcus under light microscopy contribute to fast diagnosis of $\mathrm{CM}$. The positive rate in first detection using this method was significantly higher than that using the ink- and Alcian blue-staining methods. The total positive rate was also significantly higher than that using ink staining. This method is an important auxiliary means for diagnosing CM. CSF cytological examination is a simple and easy morphological detection method. The cytological characteristics of CSF for CM include that the total cell number increases with different degrees, with mixed cell reaction or mixed lymphocyte reaction, and the proportion of eosinophil significantly increases. Therefore, excluding other intracranial parasitic infections, once the eosinophil proportion in CSF cells increases significantly, CSF examination should be conducted several times to identify cryptococcus.

\section{ACKNOWLEDGMENTS}

Research supported by the Medical Science and Technology Innovation Research Foundation of Nanjing Military Area Command (\#09MA096).

\section{REFERENCES}

Bicanic T and Harrison TS (2005). Cryptococcal meningitis. Br. Med. Bull. 72: 99-118.

Chang WN, Lu CH, Huang CR, Chuang YC, et al. (2008). Cerebrospinal fluid 14-3-3-gamma protein level in eight HIVnegative cryptococcal meningitis adults. Eur. J. Neurol. 15: 428-430.

Dammert P, Bustamante B, Ticona E, Llanos-Cuentas A, et al. (2008). Treatment of cryptococcal meningitis in Peruvian AIDS patients using amphotericin B and fluconazole. J. Infect. 57: 260-265.

He JY and He HY (2007). To investigate the early diagnosis and therapy of cryptococcal meningitis. Chin. J. Nervous Mental Dis. 33: 433-435.

Husain S, Wagener MM and Singh N (2001). Cryptococcus neoformans infection in organ transplant recipients: variables influencing clinical characteristics and outcome. Emerg. Infect. Dis. 7: 375-381.

Jain KK, Mittal SK, Kumar S and Gupta RK (2007). Imaging features of central nervous system fungal infections. Neurol. India 55: 241-250.

Juhi T, BibhaBati M, Aradhana B, Poonam L, et al. (2009). Cryptococcal meningitis in a tertiary care hospital. Nihon Ishinkin Gakkai Zasshi 50: 95-99.

Li W and Lin J (2004). Application of slide centrifugalization in detection of Cryptococcus neoformans. Chin. J. Zoonoses 20: 1100-1103.

Lin X (2009). Cryptococcus neoformans: morphogenesis, infection, and evolution. Infect. Genet. Evol. 9: $401-416$.

Macsween KF, Bicanic T, Brouwer AE, Marsh H, et al. (2005). Lumbar drainage for control of raised cerebrospinal fluid pressure in cryptococcal meningitis: case report and review. J. Infect. 51: e221-e224.

Ostxow TD and Hudgins PA (1994). Magnetic resonance imaging of intracranial fungal infections. Top. Magn. Reson. Imaging 6: 22-31. 
Pukkila-Worley R and Mylonakis E (2008). Epidemiology and management of cryptococcal meningitis: developments and challenges. Expert. Opin. Pharmacother. 9: 551-560.

Singh N, Dromer F, Perfect JR and Lortholary O (2008a). Cryptococcosis in solid organ transplant recipients: current state of the science. Clin. Infect. Dis. 47: 1321-1327.

Singh N, Lortholary O, Dromer F, Alexander BD, et al. (2008b). Central nervous system cryptococcosis in solid organ transplant recipients: clinical relevance of abnormal neuroimaging findings. Transplantation 86: 647-651.

Su MQ and Ma YY (1997). Detection of Cryptococcus neoformans by multiple polymerase chain reaction. Fourth Mil. Med. Univ. 18: 336.

Su XC and Kong FY (2001). Cytology of Cerebrospinal Fluid in Nervous System. People's Military Medical Publisher, 20-64.

Thwaites GE, Chau TT, Stepniewska K, Phu NH, et al. (2002). Diagnosis of adult tuberculous meningitis by use of clinical and laboratory features. Lancet 360: 1287-1292.

Vilchez RA, Fung J and Kusne S (2002). Cryptococcosis in organ transplant recipients: an overview. Am. J. Transplant. 2: $575-580$.

Whyte C and Eshkar N (2007). Images from headache: external hydrocephalus due to cryptococcal meningitis. Headache 47: 1447-1448.

Xue Z and Liu CC (2008). Clinical presentation and antigenic detection of cryptococcosis. J. Stroke Nervous Dis. 15: 41-44. 